\title{
Humic substances in the healthcare industry
}

Mirza M.A.

Department of Pharmaceutics, SPER, Jamia Hamdard, New Delhi-110062

doi: 10.36291/HIT.2019.mirza.014

Currently humic substances are being marketed as non-prescription products in a range of categories across the globe. Different functionalities, potentials, abundance and low cost raw material of HS augur a future in therapeutic product category or in pharmaceutical excipient. But the way forward is not as easy as in case of non-prescription products (majorly safety and quality data needed). The associated impediments are,

- requirement of full battery of safety and efficacy data (depending upon claims and categories of the products);

- availability of consistent quality raw material;

- full chemical and analytical characterization;

- low yield and consumption of by-product;

- not well recognized by regulatory agencies.

So, it requires a consorted effort by different stake holders of product development. If planned meticulously, it can also be dealt in a collaborative fashion with experts of specific fields in fine tuning with regulatory requirements. The invited talk would touch upon these nuances in details. 